\title{
Evaluación de los resultados de un programa de Dispensación Responsable de Bebidas Alcohólicas
}

\author{
Evaluation of results of a program of Responsible \\ Alcoholic Beverage Dispensing
}

\author{
J.M. Terradillos ${ }^{1}$, J.J. López-Goñi ${ }^{2}$, A. Arteaga ${ }^{2}$
}

\section{RESUMEN}

Los programas de prevención selectiva en Dispensación Responsable de Bebidas Alcohólicas (DRA) presentan diferentes evidencias de su eficacia en otros países. Sin embargo en España sólo se dispone de datos de la implementación de DRA en Barcelona. Este artículo pretende dos objetivos: valorar la efectividad de una intervención en DRA realizada en Pamplona con camareros, y evaluar los resultados tanto individual como grupalmente para identificar áreas de mejora. La muestra se compone de 40 profesionales de hostelería que participaron en alguno de los 4 cursos de DRA. Se aplicaron cuestionarios para medir pre-post conocimientos, actitudes, autoeficacia percibida y expectativas de la formación. Se presentan análisis descriptivos de todas las variables y resultados globales e individuales de la evolución de cada participante. El programa DRA llevado a cabo ofrece datos globales de mejora significativa en conocimientos, actitudes y expectativas. Los resultados encontrados evidencian la necesidad de considerar el análisis de la evolución individual de los sujetos en cada ítem.

Palabras clave. Alcohol. Formación. Prevención de riesgos. Dispensación responsable de bebidas alcohólicas. Evaluación.

\begin{abstract}
Selective prevention programs in the Responsible Dispensing of Beverages (DRA, Dispensación Responsable de Bebidas Alcohólicas) have provided varying evidence of their effectiveness in other countries. In Spain, however, data is only available for the implementation of DRA in Barcelona. This article has two aims: to assess the effectiveness of an intervention in DRA with waiters in Pamplona, and to evaluate individual and group results in order to identify areas for improvement. The sample consisted of 40 hostelry professionals who participated in one of the 4 courses of DRA. Questionnaires were used to measure pre-/post-knowledge, attitudes, perceived self-efficacy and expectations about the training. We present descriptive analyses of all the variables and individual and overall results of the evolution of each participant. The DRA program provides overall data of significant improvements in knowledge, attitudes and expectations. The results show the need to consider the analysis of the evolution of individual subjects in each item.
\end{abstract}

Key words. Alcohol. Training. Risk prevention. Responsible serving of alcoholic beverages. Evaluation.
1. Centro de Día "Aldatu" Fundación Proyecto Hombre Navarra.

2. Departamento de Psicología y Pedagogía. Universidad Pública de Navarra.

Recepción: 30 de marzo de 2011

Aceptación provisional: 24 de mayo de 2011

Aceptación definitiva: 10 de junio de 2011

\section{Correspondencia:}

Fundación Proyecto Hombre Navarra

Centro de Día "Aldatu"

Avda. Zaragoza, 23 (Edificio PP. Paúles)

31005 Pamplona

E-mail: jesus.terradillos@proyectohombrenavarra.org 


\section{INTRODUCCIÓN}

El consumo excesivo de bebidas alcohólicas es responsable de numerosos problemas en quien las consume y su entorno. Los problemas se derivan tanto de los efectos del consumo continuado a largo plazo, como de los comportamientos realizados bajo los efectos de la intoxicación. La intoxicación por alcohol está presente en uno de cada cuatro accidentes laborales $^{1}$ y en uno de cada tres accidentes mortales en carretera ${ }^{2}$. Asimismo, facilita numerosas conductas de riesgo como el consumo de otras drogas, las conductas violentas, las relaciones sexuales sin protección o la conducción temeraria ${ }^{3-7}$. Así pues, el consumo excesivo de alcohol es un factor de riesgo que conlleva diferentes problemas de salud pública. Los bares y otros locales de ocio son lugares de esparcimiento y convivencia en los que se dispensa alcohol. Las evidencias acumuladas identifican diferentes factores de riesgo para el consumo excesivo ${ }^{3,8}$ : las características físicas del local, los incentivos que establece la gerencia para el consumo, las características de la clientela y otras características externas.

El camarero, junto a los propietarios y gerentes de los locales, es el responsable del tipo de dispensación de alcohol que se hace en estos lugares. Un camarero puede ser entrenado para reconocer los signos de intoxicación en los clientes y aplicar una serie de estrategias para reducir el consumo (demorar las consumiciones, ofrecer alimentos o bebidas sin alcohol, negarse a servir) y para minimizar los riesgos del mismo (previniendo las conductas violentas y ofreciendo alternativas a la conducción $)^{3,9-12}$. Este tipo de entrenamiento se ha denominado Dispensación Responsable de Bebidas Alcohólicas (DRA) ${ }^{13}$. En cierta manera, lo que se promueve con este tipo de intervenciones es que los camareros se conviertan en agentes de salud activos, además de que se sientan y estén más capacitados para responder a diferentes situaciones problemáticas como atender $\mathrm{y}$ manejar a clientes intoxicados y negarse a servir alcohol a menores de edad. Esta perspectiva es útil para superar la contradicción en que se encuentran los propios camareros y sus empleadores, que han de decidir entre el "negocio" de la dispensación y la responsabilidad para con sus clientes $^{14}$.

Estas intervenciones son comunes en Estados Unidos y en el norte de Europa ${ }^{13}$, pero en nuestro entorno todavía no se han generalizado $^{3,9,13}$, aunque forman parte de la estrategia mundial para reducir el uso nocivo del alcohol ${ }^{10}$. Los cursos de DRA suelen variar en su duración, contenidos, estrategias y destinatarios. La duración mínima para que sean eficaces es de 4 horas ${ }^{15}$. Se suelen organizar en distintos contextos de formación y aunque la evidencia científica acerca de los resultados de dichos programas va en aumento ${ }^{3}$, se necesita seguir investigando ${ }^{12,16,17}$.

A pesar de las diferentes evidencias de la efectividad de la DRA encontradas en otros países, los resultados encontrados hasta la fecha en España son desalentadores. En la evaluación de un programa de DRA realizado en Barcelona con camareros no se encontraron mejorías estadísticamente significativas en conocimientos, actitudes o autoeficacia percibida ${ }^{13}$. Esta falta de mejoría se atribuyó, en parte, a que la muestra estaba compuesta por camareros con una amplia experiencia en el sector y con buenos conocimientos de partida. Los autores supusieron que a mayor conocimiento del problema, y a mayor experiencia, se daba una mayor conciencia de las propias limitaciones y por ello una mayor dificultad para adquirir estrategias preventivas en la dispensación de alcohol. A las dos dificultades anteriores, quizá se pueda unir una tercera de carácter metodológico. Para la evaluación de la intervención se empleó una comparación entre las medidas iniciales y finales, considerando a todos los participantes como un único grupo. Este análisis puede enmascarar la evolución de cada uno de los participantes ya que puede darse el caso de que unos sujetos mejorasen, otros empeorasen, y otros siguiesen igual. Si se tuviera en cuenta la evolución de cada participante se podría afinar mucho más en 
las estrategias de intervención ${ }^{18}$. Por esta razón, dos son los objetivos de la presente investigación: 1. Valorar la efectividad de una intervención de DRA realizada en Pamplona con camareros; y 2. Realizar una evaluación de los resultados tanto individual como grupal para poder identificar áreas de mejora de la intervención en DRA.

\section{MATERIAL Y MÉTODOS}

\section{Participantes}

La muestra está compuesta por 40 profesionales de la hostelería de 30 establecimientos diferentes que acuden a uno de los cuatro cursos de formación. La edad media es de 33,6 años (D.T.=9,8); 32 de ellos son mujeres (80\%) con una edad media de 33,1 años (D.T $=9,6$ ) frente a los 35,6 años de media (D.T.=11,1) de los hombres. La experiencia media de trabajo en el sector es de 2,7 años (D.T.=5,4).

Dado el carácter formativo de la actividad, los criterios de inclusión fueron: ser profesionales de la hostelería y matricularse en el curso. No se planteó ningún criterio de exclusión. Todos los camareros que solicitaron participar en el curso fueron admitidos. Todos ellos $(\mathrm{n}=40)$ finalizaron la formación y cumplimentaron los instrumentos de evaluación.

\section{Intervención}

Se trata de un programa de prevención, adaptado por los técnicos de la Fundación Proyecto Hombre Navarra a partir del Programa de Dispensación Responsable de la Agencia de Salud Pública de Barcelona diseñado por Rodríguez-Martos y $\mathrm{col}^{13}$. El programa se presenta en un curso de 4 horas de duración, y los contenidos que se incluyen son los siguientes: 1. Aspectos legales de la dispensación de alcohol; 2. Información sobre las bebidas alcohólicas y sus efectos (con especial énfasis en el concepto de Unidad de Bebida Estándar -UBE- ya que ayuda al profesional de hostelería a tener más información del nivel de intoxicación del cliente, así como a estimar aproximadamente el tiempo de eliminación de las cantidades de alcohol consumidas); 3. Estrategias de manejo de situaciones (no servir alcohol a menores o a clientes ebrios y ofrecer alternativas a la conducción); y 4. Habilidades de comunicación y de resolución de conflictos para la prevención de conductas violentas.

\section{Instrumentos de evaluación}

El Cuestionario de Conocimientos, Actitudes, Autoeficacia Percibida y Expectativas de la formación en DRA ${ }^{13}$ consta de 20 cuestiones de respuesta cerrada verdadero-falso, y está diseñado para valorar la evolución de cada participante entre el pre y el post en cada una de las áreas señaladas. En concreto, hay 7 preguntas que valoran los conocimientos de los participantes. El rango de puntuaciones varía entre 0 (ninguna respuesta correcta) y 7 (todas las respuestas correctas). Hay 5 preguntas que evalúan las actitudes de los sujetos, con lo que el rango de puntuaciones varía entre 0 (nula actitud hacia la DRA) y 5 (actitud positiva hacia la DRA). La percepción de autoeficacia en la DRA es valorada por 4 preguntas. El rango de puntuaciones oscila entre 0 (ninguna sensación de autoeficacia) y 4 (percepción completa de autoeficacia). Por último, las expectativas en la formación del DRA están valoradas por 4 preguntas, con un rango que oscila entre 0 (nula expectativa) y 4 (alta expectativa en la efectividad de la DRA).

El Cuestionario de Conocimientos sobre el Alcohol de Terradillos y Arteaga ${ }^{19}$ es un instrumento realizado ad hoc de 25 ítems de respuesta dicotómica (verdaderofalso) que valora los conocimientos sobre el alcohol. Más concretamente valora una serie de creencias irracionales sobre los efectos del alcohol. El rango de puntuaciones obtenidas varía entre 0 (ninguna respuesta correcta) y 25 (todas las respuestas correctas). Se emplea como medida de resultados de los conocimientos adquiridos y como complemento al cuestionario descrito anteriormente. 
El Cuestionario de Valoración de la Actividad es un cuestionario creado ad hoc de 24 preguntas en formato Likert (de 5 alternativas de respuesta, con valores entre $1 \mathrm{y}$ 5) que valora la satisfacción de los participantes en 7 apartados relacionados con el curso: información previa a la actividad, contenidos del curso, metodología seguida, calidad de la documentación entregada, calidad del profesorado, organización de los tiempos y resultados de la actividad. Con el objetivo de disminuir el efecto de la deseabilidad sobre las respuestas, este cuestionario se respondió de forma anónima.

\section{Procedimiento}

Tras la preparación y adaptación de los contenidos del curso teniendo en cuenta la legislación foral navarra, la Fundación Proyecto Hombre Navarra, en colaboración con el Ayuntamiento de Pamplona y con la Asociación de Hostelería de Navarra, organizó entre 2006 y 2009 cuatro cursos de DRA de cuatro horas de duración cada uno, dirigidos a empleados de hostelería. La participación de los camareros fue voluntaria y como incentivo para los hosteleros, el área de Servicios Sociales del Ayuntamiento de Pamplona realizó un distintivo que podrían exhibir los locales que hubieran enviado al menos a un camarero a los cursos. Además se dio a conocer en los medios de comunicación de ámbito local y nacional qué locales habían participado en el programa.

Al comienzo del curso se aplicaron el Cuestionario de Conocimientos, Actitudes, Autoeficacia Percibida y Expectativas de la formación en DRA y el Cuestionario de Conocimientos sobre el Alcohol de Terradillos y Arteaga. Posteriormente, tras realizar las 4 horas de formación se volvieron a pasar los dos cuestionarios anteriores junto con el Cuestionario de Valoración de la Actividad. Por último, se proporcionaron a los participantes las respuestas correctas del cuestionario de conocimientos junto con las argumentaciones.

\section{Análisis estadístico}

En primer lugar se realizaron análisis descriptivos para todas las variables. En segundo lugar se valoró la ejecución pretest-postest desde una perspectiva grupal. Para ello se realizó una comparación de medias a partir de un diseño de medidas repetidas test-retest mediante la prueba no paramétrica de Wilcoxon de los rangos con signo. En tercer lugar se realizó un análisis individual de la evolución de cada asistente. Para ello se valoró la ejecución de cada participante en cada uno de los ítems tanto en el pretest como en el postest. Desde esta perspectiva, y dado que cada ítem tenía una única respuesta "correcta" o "adecuada", las posibilidades de corrección eran 4: 1. El participante mejora (mala respuesta pre-test y buena respuesta post-test); 2 . Empeora (buena respuesta pre-test y mala respuesta post-test); 3 . Sigue equivocado (mala respuesta pretest y postest); o 4 . Sigue acertado (buena respuesta pretest y postest). Para cada uno de los subapartados se hizo un recuento de cada una de las posibilidades, permitiendo una valoración global de los ítems. En este caso también se empleó como contraste de hipótesis la prueba no paramétrica de Wilcoxon. Todos los análisis de datos se realizaron mediante el programa estadístico SPSS vs. 15.0.

\section{RESULTADOS}

\section{Resultados globales de la intervención}

En la tabla 1 se presenta la diferencia de medias entre los momentos previo y posterior a la intervención, así como los resultados que obtuvo en su día Rodríguez-Martos (2004).

Desde una perspectiva grupal se observa que, tanto en los conocimientos valorados con el cuestionario de Terradillos y Arteaga ${ }^{19}$, como en las actitudes y expectativas de los participantes evaluados con el cuestionario de Rodríguez-Martos y col se produce una mejoría estadísticamente significativa tras la intervención en DRA. 
Tabla 1. Diferencia de medias entre el post y el pre-curso en las diferentes pruebas junto con los resultados de Rodríguez-Martos y col (2004)

\begin{tabular}{|c|c|c|c|c|c|c|c|c|}
\hline & & \multicolumn{5}{|c|}{$(N=40)$} & \multicolumn{2}{|c|}{$\begin{array}{c}\text { Rodríguez-Martos } \\
\text { y col, 2004 } \\
\text { (N = 24) }\end{array}$} \\
\hline & & & & & & & Pretest & Postest \\
\hline \multicolumn{2}{|l|}{ Cuestionario } & Media & D.T. & Media & D.T. & & Media & Media \\
\hline $\begin{array}{l}\text { (Terradillos- } \\
\text { Arteaga, } \\
\text { 2006) }\end{array}$ & Conocimiento & 18,0 & 2,3 & 19,0 & 2,4 & $3,9 * * *$ & - & - \\
\hline \multirow{4}{*}{$\begin{array}{l}\text { (Rodríguez- } \\
\text { Martos y col, } \\
\text { 2004) }\end{array}$} & Conocimiento & 5,4 & 1,1 & 5,6 & 0,9 & 0,6 & 5,3 & 5,7 \\
\hline & Actitud & 4,1 & 0,9 & 4,6 & 0,5 & $3,0^{* *}$ & 3,9 & 4,2 \\
\hline & Autoeficacia & 1,9 & 1,2 & 2,2 & 1,2 & 1,6 & 2,7 & 2,6 \\
\hline & Expectativas & 2,9 & 1,5 & 3,6 & 1,0 & $2,6^{*}$ & 1,6 & 2,2 \\
\hline
\end{tabular}

${ }^{*} p<0,05 ;{ }^{* *} p<0,01 ;{ }^{* *} p<0,001$

\section{Resultados individuales en el cuestionario de Rodríguez-Martos y colaboradores}

Desde una perspectiva individual, el $55 \%$ de los asistentes mejoró la puntuación en el postest respecto al pretest, el $30 \%$ empeoró y el $15 \%$ mantuvo su puntuación. En la tabla 2 se presentan los resultados de la evolución en los diferentes ítems de los participantes en el curso. En dicha tabla se aprecian las cuestiones en las que algunos participantes empeoraron sus conocimientos o actitudes.

Tanto en el ítem 1 como en el 5 del apartado de conocimientos, que valoran aspectos sobre el contenido y la eliminación del alcohol, existe un porcentaje significativo de participantes que empeoraron su resultado respecto a las puntuaciones obtenidas en el pretest. Sin embargo, en el ítem 2 , referido a la interacción entre café y alcohol, mejoraron significativamente.
En cuanto a las actitudes, existe una mejoría estadísticamente significativa en 4 de los 5 ítems. Sin embargo, tras realizar el curso un $22,5 \%(n=9)$ de los participantes pasó de creer que el camarero debe intervenir sobre la decisión de consumo de los clientes a creer que no, y un $17,5 \%(\mathrm{n}=7)$ mantuvo la creencia de que no se debe intervenir sobre la decisión de consumo de los clientes.

Respecto a la autoeficacia percibida, un $17,5 \%$ de los participantes ( $n=7$ ) pasó de considerarse capaz de negarse a servir alcohol a un cliente ebrio a considerarse incapaz. Otro $32,5 \%(\mathrm{n}=13)$ mantuvo su incapacidad inicial. En cuanto a la habilidad de detectar documentos de identidad falsos, un $10 \%$ de los asistentes $(n=4)$, pasaron de considerarse capaces a considerarse incapaces y el $55 \%(n=22)$ mantuvieron su incapacidad inicial. No aparecen diferencias significativas en las expectativas de los participantes respecto a los efectos de la Dispensación Responsable de Bebidas Alcohólicas. 
Tabla 2. Evolución de los participantes en el curso ítem por ítem

\begin{tabular}{|c|c|c|c|c|c|c|c|c|c|}
\hline & \multicolumn{2}{|c|}{ Empeoran } & \multicolumn{2}{|c|}{$\begin{array}{l}\text { Mantienen } \\
\text { negativa }\end{array}$} & \multicolumn{2}{|c|}{$\begin{array}{l}\text { Mantienen } \\
\text { positiva }\end{array}$} & \multicolumn{2}{|c|}{ Mejoran } & \multirow[t]{2}{*}{ Wilcoxon } \\
\hline & $\mathbf{n}$ & (\%) & $\mathbf{n}$ & (\%) & $\mathbf{n}$ & (\%) & $\mathbf{n}$ & (\%) & \\
\hline \multicolumn{10}{|l|}{ Conocimientos } \\
\hline $\begin{array}{l}\text { 1. ¿Una copa de coñac tiene el mismo } \\
\text { contenido de alcohol que una } \\
\text { cerveza mediana? }\end{array}$ & 8 & $(20,0 \%)$ & 0 & - & 30 & $(75,0 \%)$ & 2 & $(5,0 \%)$ & $2,7^{* *}$ \\
\hline $\begin{array}{l}\text { 2. ¿Una taza de café cargado ayuda a } \\
\text { eliminar los efectos del alcohol que } \\
\text { se ha bebido? }\end{array}$ & 0 & - & 0 & - & 27 & $(67,5 \%)$ & 13 & $(32,5 \%)$ & $3,6 * * *$ \\
\hline $\begin{array}{l}\text { 3. ¿Es el alcohol la principal droga } \\
\text { de abuso en España? (en cuanto a } \\
\text { número de consumidores) }\end{array}$ & & & 1 & $(2,5 \%)$ & 37 & $(92,5 \%)$ & 2 & $(5,0 \%)$ & 0,6 \\
\hline $\begin{array}{l}\text { 4. ¿Es legal servir alcohol a menores de } \\
18 \text { años (en Navarra)? }\end{array}$ & 4 & $(10,0 \%)$ & 1 & $(2,5 \%)$ & 31 & $(77,5 \%)$ & 4 & $(10,0 \%)$ & 1,3 \\
\hline $\begin{array}{l}\text { 5. ¿Para eliminar los efectos de } 2 \\
\text { «cubatas» es necesario que pasen } 2 \\
\text { horas? }\end{array}$ & 20 & $(50,0 \%)$ & 9 & $(22,5 \%)$ & 9 & $(22,5 \%)$ & 2 & $(5,0 \%)$ & $4,8^{* * *}$ \\
\hline $\begin{array}{l}\text { 6. ¿Sabes si } 2 \text { vasos de vino son } \\
\text { suficientes para que un adulto de } \\
\text { peso medio dé positivo }(0,5) \text { en un } \\
\text { control de alcoholemia? }\end{array}$ & 1 & $(2,5 \%)$ & 6 & $(15,0 \%)$ & 25 & $(62,5 \%)$ & 8 & $(20,0 \%)$ & 0 \\
\hline $\begin{array}{l}\text { 7. ¿El límite legal de alcoholemia para } \\
\text { los conductores profesionales es de } \\
0.3 \text { ? }\end{array}$ & 2 & $(5,0 \%)$ & 6 & $(15,0 \%)$ & 20 & $(50,0 \%)$ & 12 & $(30,0 \%)$ & 0,4 \\
\hline \multicolumn{10}{|l|}{ Actitudes } \\
\hline $\begin{array}{l}\text { 1. ¿Crees que el personal de un } \\
\text { bar puede ayudar a reducir } \\
\text { las consecuencias negativas } \\
\text { relacionadas con el consumo de } \\
\text { alcohol? }\end{array}$ & 0 & - & 0 & - & 31 & $(77,5 \%)$ & 9 & $(22,5 \%)$ & $3,0^{* *}$ \\
\hline $\begin{array}{l}\text { 2. ¿Crees que el personal de un bar } \\
\text { puede animar a los clientes a que } \\
\text { beban con moderación? }\end{array}$ & 0 & - & 0 & - & 35 & $(87,5 \%)$ & 5 & $(12,5 \%)$ & $2,2^{*}$ \\
\hline $\begin{array}{l}\text { 3. ¿Crees que el personal de un bar } \\
\text { debería comprobar la edad de los } \\
\text { clientes jóvenes pidiendo el DNI } \\
\text { antes de servirles alcohol? }\end{array}$ & 0 & - & 0 & - & 38 & $(95,0 \%)$ & 2 & $(5,0 \%)$ & 1,4 \\
\hline $\begin{array}{l}\text { 4. ¿Crees que el personal de un bar no } \\
\text { debe entrometerse en la decisión } \\
\text { del cliente sobre su consumo de } \\
\text { alcohol? }\end{array}$ & 9 & $(22,5 \%)$ & 7 & $(17,5 \%)$ & 13 & $(32,5 \%)$ & 11 & $(27,5 \%)$ & $2,1^{*}$ \\
\hline $\begin{array}{l}\text { 5. ¿Crees que el personal de un bar } \\
\text { debería apoyar el mensaje de "si } \\
\text { bebes no conduzcas" intentando } \\
\text { influir sobre sus clientes al respecto? }\end{array}$ & 0 & - & 0 & - & 36 & $(90,0 \%)$ & 4 & $(10,0 \%)$ & $2,0^{*}$ \\
\hline
\end{tabular}




\begin{tabular}{|c|c|c|c|c|c|c|c|c|c|}
\hline & \multicolumn{2}{|c|}{ Empeoran } & \multicolumn{2}{|c|}{$\begin{array}{l}\text { Mantienen } \\
\text { negativa }\end{array}$} & \multicolumn{2}{|c|}{$\begin{array}{l}\text { Mantienen } \\
\text { positiva }\end{array}$} & \multicolumn{2}{|c|}{ Mejoran } & \multirow[t]{2}{*}{ Wilcoxon } \\
\hline & $\mathbf{n}$ & (\%) & $\mathbf{n}$ & (\%) & $\mathbf{n}$ & (\%) & $\mathbf{n}$ & (\%) & \\
\hline \multicolumn{10}{|l|}{ Autoeficacia } \\
\hline $\begin{array}{l}\text { 1. ¿Te resulta difícil saber cuándo una } \\
\text { persona está intoxicada (borracha)? }\end{array}$ & 2 & $(5,0 \%)$ & 3 & $(7,5 \%)$ & 31 & $(77,5 \%)$ & 4 & $(10,0 \%)$ & 0,8 \\
\hline $\begin{array}{l}\text { 2. ¿Te resulta difícil negarte a servir a } \\
\text { una persona ebria? }\end{array}$ & 7 & $(17,5 \%)$ & 13 & $(32,5 \%)$ & 15 & $(37,5 \%)$ & 5 & $(12,5 \%)$ & $3,2^{* *}$ \\
\hline $\begin{array}{l}\text { 3. ¿Te resulta difícil detectar si un DNI } \\
\text { es falso? }\end{array}$ & 4 & $(10,0 \%)$ & 22 & $(55,0 \%)$ & 9 & $(22,5 \%)$ & 5 & $(12,5 \%)$ & $3,8^{* * *}$ \\
\hline $\begin{array}{l}\text { 4. ¿Te resulta difícil hacer que un } \\
\text { cliente no conduzca tras haber } \\
\text { bebido? }\end{array}$ & 2 & $(5,0 \%)$ & 20 & $(50,0 \%)$ & 6 & $(15,0 \%)$ & 12 & $(30,0 \%)$ & 1,9 \\
\hline \multicolumn{10}{|l|}{ Expectativas } \\
\hline $\begin{array}{l}\text { 1. ¿Crees que la DRA ayudará a } \\
\text { reducir el número de clientes que se } \\
\text { embriagan? }\end{array}$ & 2 & $(5,0 \%)$ & 2 & $(5,0 \%)$ & 29 & $(72,5 \%)$ & 7 & $(17,5 \%)$ & 0,2 \\
\hline $\begin{array}{l}\text { 2. ¿Crees que la DRA ayudará a reducir } \\
\text { el número de menores que beben } \\
\text { alcohol? }\end{array}$ & 4 & $(10,0 \%)$ & 3 & $(7,5 \%)$ & 25 & $(62,5 \%)$ & 8 & $(20,0 \%)$ & 0,7 \\
\hline $\begin{array}{l}\text { 3. ¿Crees que la DRA disminuirá el } \\
\text { número de personas que beben y } \\
\text { conducen un vehículo? }\end{array}$ & 1 & $(2,5 \%)$ & 1 & $(2,5 \%)$ & 29 & $(72,5 \%)$ & 9 & $(22,5 \%)$ & 0,1 \\
\hline $\begin{array}{l}\text { 4. ¿Crees que la DRA disminuirá las } \\
\text { conductas violentas y los accidentes } \\
\text { de tráfico relacionados con el } \\
\text { alcohol? }\end{array}$ & 2 & $(5,0 \%)$ & 2 & $(5,0 \%)$ & 25 & $(62,5 \%)$ & 11 & $(27,5 \%)$ & 0,3 \\
\hline
\end{tabular}

${ }^{*} p<0,05 ;{ }^{* *} p<0,001$

\section{Resultados individuales en el cuestionario de Terradillos y Arteaga}

En el análisis ítem por ítem (Tabla 3) se aprecia que en 15 de los ítems hubo una disminución de los errores cometidos antes y después de la formación, frente a 6 ítems que empeoraron. El ítem en el que más se empeoró es el número 22 , que se refiere al cálculo de la cantidad de alcohol que contiene una bebida alcohólica. En él se dio un aumento del 20\% (8 sujetos) de errores en las respuestas postest. El $50 \%$ de los sujetos $(n=20)$ erraron la respuesta de dicho ítem antes de la intervención frente al $70 \%$ de los participantes $(n=28)$ que lo hicieron posteriormente. 
Tabla 3. Resultados de modificación de ideas erróneas sobre el alcohol

\begin{tabular}{|c|c|c|c|c|c|}
\hline item & $\begin{array}{c}\text { Cuestionario de Conocimientos sobre el alcohol } \\
\text { Terradillos-Arteaga (2006) }\end{array}$ & $\begin{array}{l}\text { Errores } \\
\text { pre }\end{array}$ & $\begin{array}{c}\text { Errores } \\
\text { post }\end{array}$ & $\begin{array}{l}\text { Diferencia } \\
\text { de errores }\end{array}$ & \\
\hline 2 & $\begin{array}{l}\text { Tomar café es una buena manera de recuperar la } \\
\text { sobriedad si estás bebido }\end{array}$ & $12(30 \%)$ & $2(5 \%)$ & $-10(-25 \%)$ & \multirow{15}{*}{ 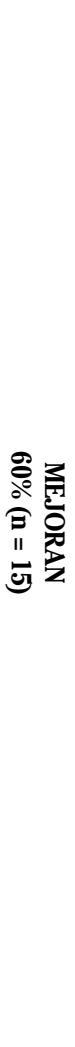 } \\
\hline 15 & $\begin{array}{l}\text { Bebiendo lo mismo, la alcoholemia sube más en la mujer } \\
\text { que en el hombre }\end{array}$ & $12(30 \%)$ & $5(12,5 \%)$ & $-7(-17,5 \%)$ & \\
\hline 16 & $\begin{array}{l}\text { Las bebidas alcohólicas diluidas (con agua o refresco) } \\
\text { "se suben menos a la cabeza" }\end{array}$ & $17(42,5 \%)$ & $10(25 \%)$ & $-7(-17,5 \%)$ & \\
\hline 1 & El alcohol va bien para curar resfriados & $7(17,5 \%)$ & $1(2,5 \%)$ & $-6(-15 \%)$ & \\
\hline 12 & $\begin{array}{l}\text { Un poco de "quina" (vino dulce) es indicada para niños } \\
\text { que han perdido el apetito }\end{array}$ & $13(32,5 \%)$ & $8(20 \%)$ & $-5(-12,5 \%)$ & \\
\hline 23 & El alcohol es un estimulante & $29(72,5 \%)$ & $24(60 \%)$ & $-5(-12,5 \%)$ & \\
\hline 14 & $\begin{array}{l}\text { Es peor para la salud beber alcohol en ayunas que } \\
\text { beberlo en las comidas }\end{array}$ & $6(15 \%)$ & $2(5 \%)$ & $-4(-10 \%)$ & \\
\hline 17 & $\begin{array}{l}\text { La gente que bebe mucho alcohol incrementa la potencia } \\
\text { sexual }\end{array}$ & $9(22,5 \%)$ & $5(12,5 \%)$ & $-4(-10 \%)$ & \\
\hline 9 & $\begin{array}{l}\text { El alcohol hace que sobrestimes tus facultades cuando } \\
\text { conduces }\end{array}$ & $9(22,5 \%)$ & $7(17,5 \%)$ & $-2(-5 \%)$ & \\
\hline 13 & $\begin{array}{l}\text { Un bebedor "de fin de semana" no puede llegar a ser } \\
\text { alcohólico }\end{array}$ & $9(22,5 \%)$ & $7(17,5 \%)$ & $-2(-5 \%)$ & \\
\hline 6 & El uso moderado de alcohol hace mejorar los reflejos & $2(5 \%)$ & $1(2,5 \%)$ & $-1(-2,5 \%)$ & \\
\hline 21 & $\begin{array}{l}\text { Para pasárselo realmente bien, es imprescindible ir "un } \\
\text { poco colocado" }\end{array}$ & $2(5 \%)$ & $1(2,5 \%)$ & $-1(-2,5 \%)$ & \\
\hline 5 & $\begin{array}{l}\text { La cerveza 'sin alcohol”, contiene algo de alcohol (menos } \\
\text { de un grado) }\end{array}$ & $7(17,5 \%)$ & $6(15 \%)$ & $-1(-2,5 \%)$ & \\
\hline 11 & El alcohol ayuda a desinhibirse sexualmente & $12(30 \%)$ & $11(27,5 \%)$ & $-1(-2,5 \%)$ & \\
\hline 24 & Hay más hombres alcohólicos que mujeres alcohólicas & $15(37,5 \%)$ & $14(35 \%)$ & $-1(-2,5 \%)$ & \\
\hline 18 & El alcohol disminuye la agudeza visual & $3(7,5 \%)$ & $3(7,5 \%)$ & $0(0 \%)$ & \multirow{4}{*}{ 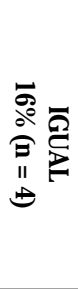 } \\
\hline 8 & $\begin{array}{l}\text { El alcohol mejora la capacidad creativa, lo demuestra el } \\
\text { consumo excesivo de muchos artistas }\end{array}$ & $6(15 \%)$ & $6(15 \%)$ & $0(0 \%)$ & \\
\hline 25 & $\begin{array}{l}\text { Los hijos de mujeres bebedoras pesan un } 15 \% \text { menos al } \\
\text { nacer que los de mujeres no-bebedoras }\end{array}$ & $8(20 \%)$ & $8(20 \%)$ & $0(0 \%)$ & \\
\hline 20 & $\begin{array}{l}\text { El alcohol engorda (aunque los alcohólicos suelen estar } \\
\text { delgados) }\end{array}$ & $13(32,5 \%)$ & $13(32,5 \%)$ & $0(0 \%)$ & \\
\hline 7 & $\begin{array}{l}\text { No se puede desarrollar una dependencia alcohólica sólo } \\
\text { por beber cerveza }\end{array}$ & $11(27,5 \%)$ & $12(30 \%)$ & $1(2,5 \%)$ & \multirow{6}{*}{ 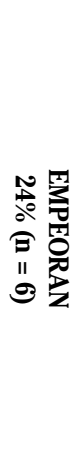 } \\
\hline 19 & $\begin{array}{l}\text { El alcohol cura los sentimientos de inferioridad, timidez } \\
\text { o vergüenza }\end{array}$ & $14(35 \%)$ & $15(37,5 \%)$ & $1(2,5 \%)$ & \\
\hline 3 & $\begin{array}{l}\text { El uso moderado de alcohol mejora la presión arterial de } \\
\text { quienes la tienen baja }\end{array}$ & $21(52,5 \%)$ & $22(55 \%)$ & $1(2,5 \%)$ & \\
\hline 4 & $\begin{array}{l}\text { El cava (o champán) se "sube más a la cabeza" que el } \\
\text { vino ordinario }\end{array}$ & $25(62,5 \%)$ & $26(65 \%)$ & $1(2,5 \%)$ & \\
\hline 10 & $\begin{array}{l}\text { En Navarra está prohibida la venta de bebidas } \\
\text { alcohólicas a menores de } 18 \text { años }\end{array}$ & $0(0 \%)$ & $2(5 \%)$ & $2(5 \%)$ & \\
\hline 22 & $\begin{array}{l}\text { Una lata de cerveza contiene la mitad de alcohol que una } \\
\text { copa de coñac }\end{array}$ & $20(50 \%)$ & $28(70 \%)$ & $8(20 \%)$ & \\
\hline
\end{tabular}




\section{Cuestionario de valoración de la actividad}

Tal y como se presenta en la tabla 4 , los alumnos del curso valoran con una media por encima de 4 (en un rango de 1 a 5) to- dos los aspectos relacionados con la calidad del curso: información previa sobre el curso, contenidos tratados, metodología utilizada, documentación entregada, organización y resultados.

Tabla 4. Resultados del cuestionario de valoración de la actividad

\begin{tabular}{lcccc}
\hline \multicolumn{1}{c}{$\mathbf{N}=\mathbf{4 0}$} & Mínimo & Máximo & Media & Desv. típ. \\
\hline Profesorado (1: Muy bajo; 5: Excelente) & 3,0 & 5,0 & 4,7 &, 5 \\
\hline Organización (1: Malo; 5: Excelente) & 2,0 & 5,0 & 4,4 &, 7 \\
\hline Contenido (1: Muy poco adecuado; 5: Muy adecuado) & 2,0 & 5,0 & 4,3 &, 6 \\
\hline Metodología (1: Muy poco adecuado; 5: Muy adecuado) & 3,0 & 5,0 & 4,3 &, 7 \\
\hline Documentación (1: Malo; 5: Excelente) & 2,0 & 5,0 & 4,3 &, 8 \\
\hline Información Previa (1: Deficiente; 5: Excelente) & 2,0 & 5,0 & 4,1 &, 9 \\
\hline Resultados (1: Muy bajo; 5: Muy alto) & 1,0 & 5,0 & 4,1 &, 8 \\
\hline
\end{tabular}

\section{DISCUSIÓN}

El programa de formación en DRA llevado a cabo en este estudio ofrece datos de mejoras significativas en los conocimientos, actitudes y expectativas de algunos de los profesionales de hostelería, en un contexto en el que han colaborado los propios hosteleros y una administración pública. Estos resultados son los primeros que avalan la efectividad de este tipo de intervenciones en nuestro entorno, y justifican continuar con esta línea de investigación para poder mejorar y adecuar los contenidos a los diferentes perfiles de sujetos. Además sitúan este tipo de intervención dentro de los resultados efectivos encontrados en otros entornos culturales ${ }^{3,13,15}$ favoreciendo la compatibilidad de la responsabilidad de los hosteleros con el propio negocio ${ }^{14}$. Con estos datos también se justifica el diseño de intervenciones específicas que valoren la eficiencia de este tipo de programas. Sólo en Pamplona se estima que hay unos 1.000 establecimientos que dispensan alcohol, y cuyo personal podría ser formado en estas prácticas responsables.

En cuanto a los conocimientos presentados durante la intervención, se aprecia en los sujetos un cambio significativo en las ideas erróneas sobre los efectos del alcohol. Al realizar el análisis de resultados ítem por ítem (Tabla 3), se puede observar que se da una mejoría en el $60 \%$ de los ítems ( 15 casos), en el $16 \%$ no se modifica ( 4 casos) y empeora en el $24 \%$ (6 casos). El único ítem que empeora de una forma estadísticamente significativa es el referido al cálculo de las UBE. Lo mismo ocurre en el ítem correspondiente del cuestionario de Rodríguez-Martos (2004). Quizá el propio concepto de UBE, aunque útil para facilitar el registro de consumo alcohólico ${ }^{20,21}$, sea lo suficientemente complejo como para necesitar de una mayor atención en el curso, y por ello quizá la intervención debería ser mayor de 4 horas. En cualquier caso, parece interesante la utilización del cuestionario de Terradillos y Arteaga ${ }^{19}$ para la valoración de las ideas irracionales sobre el alcohol, sobre todo si se tiene en cuenta su rápida y fácil aplicación junto con las posibilidades que brinda comentar las respuestas con el grupo participante en la intervención. Además hay que considerar que así como las creencias irracionales sobre el alcohol facilitan su consumo abusi- 
vo ${ }^{22}$, también podrían estar en la base de un comportamiento inadecuado de los camareros con sus clientes.

Algunos autores también han sugerido la idoneidad de ampliar la formación de los camareros a la detección de señales de intoxicación en los clientes tanto de alcohol ${ }^{11}$ como de otras drogas ${ }^{3}$. Es otra sugerente línea de investigación, ya que en el desarrollo del curso surgieron cuestiones relativas a la intoxicación con otras sustancias, no sólo el alcohol. De hecho, los camareros tenían la percepción de una mayor agresividad y dificultad de manejo con los clientes bajo los efectos simultáneos del alcohol y de estimulantes como la cocaína. Diferentes investigaciones avalan esta percepción ${ }^{14,23,24}$ y por ello se debe valorar la inclusión de conocimientos sobre esta cuestión.

En el apartado de actitudes se observa un empeoramiento en el ítem referente a la intromisión del profesional en la decisión del cliente sobre su consumo. Cabe señalar que dicho ítem está formulado en negativo, lo cual nos podría indicar una mayor dificultad en los participantes para responder adecuadamente. También se puede pensar que el aumento de conciencia sobre la dificultad de manejo en esta situación influye en un empeoramiento del resultado y que debería entenderse como un cambio hacia una actitud más realista que la inicial. En todo caso, otra línea de investigación futura está relacionada con mejorar las propiedades psicométricas de los instrumentos utilizados.

En cuanto a la autoeficacia percibida, desde una perspectiva grupal no aparecen diferencias estadísticamente significativas. Ahora bien, al realizar el análisis individual se comprueba que, tras completar el curso, hay más personas a las que les resulta más difícil detectar un DNI falso o negarse a servir a una persona ebria. Sin duda, estos aspectos han de mejorarse en futuras intervenciones puesto que ambas son habilidades que se pueden entrenar.

Desde una perspectiva global, los resultados obtenidos evidencian la necesidad de considerar la evolución de cada alumno en cada uno de los ítems propuestos. Este tipo de análisis ofrece datos concretos para mejorar los contenidos de la actividad y dan una visión más detallada de los resultados, resaltando que se da una mejoría en cuanto a conocimientos, actitudes y expectativas de autoeficacia de los participantes. A partir de ahora, además de continuar con esta estrategia de formación, se pueden diseñar nuevas intervenciones para valorar el posterior comportamiento en el puesto de trabajo de los asistentes a este tipo de cursos.

En cuanto a la valoración que hacen los alumnos del curso, ésta es, en general, muy buena. Además hay que resaltar el hecho de que nadie abandonó el curso antes de su finalización. Las dos valoraciones más bajas se refieren a la información previa del curso y a los resultados del mismo. A pesar de ello, la valoración global en estas cuestiones es de 4 (buena-adecuada) sobre 5. Parece que hay poco margen de mejora en los aspectos valorados, por lo que el esfuerzo debería centrarse en la adecuación de los contenidos y en valorar los efectos de la formación.

El presente estudio presenta algunas limitaciones, por lo que hay que ser cautos en la generalización de los resultados obtenidos. Quizá la mayor limitación de este estudio sea que no dispone de datos para valorar si ha habido mejoría en el comportamiento de los camareros tras el curso. En el futuro deberían emplearse también otro tipo de medidas de evaluación más ecológicas, tanto para los conocimientos como para las actitudes y la autoeficacia percibida, así como sobre el comportamiento posterior, tal y como se han empleado en otros estudios $^{11,13}$. Otra limitación se relaciona con la composición de la muestra. Ciertamente es una muestra pequeña, por lo que hay que ser muy prudentes antes de generalizar los resultados obtenidos. Por otro lado, hay que tener presente que la muestra se ha conformado con camareros voluntarios. Esta voluntariedad puede explicar los altos niveles de expectativas entre el alumnado. Una futura línea de investigación puede ser el efecto de la inclusión de estos contenidos en los programas de formación reglada $u$ obligatorios. Quizá en esos casos se encuentren unas mayores diferencias entre los resultados antes y después de la intervención. 


\section{Agradecimientos}

Los autores desean agradecer a la Asociación de Hostelería de Navarra y al Ayuntamiento de Pamplona por su ayuda para la realización de la presente investigación. Asimismo, desean agradecer a la Dra. Alicia Rodríguez Martos-Dauer por su disponibilidad y colaboración para la realización de la intervención, y al Dr. Javier Fernández-Montalvo la inestimable ayuda prestada para la elaboración del presente manuscrito.

\section{BIBLIOGRAFÍA}

1. Bastida N. Problemas laborales asociados al consumo de alcohol. Adicciones 2002; 14 : 239-251.

2. Instituto Nacional de Toxicología y Ciencias Forenses. Memoria análisis toxicológico muertes en accidentes de tráfico 2008. Madrid: Ministerio de Justicia 2009.

3. Miller BA, Holder HD, VoAs RB. Environmental strategies for prevention of drug use and risks in clubs. J Subst Use 2009; 14: 19-38.

4. Revuelta E, Godoy P, Farreny M. Evolución de la mortalidad atribuible al consumo de alcohol en Cataluña, 1988-1997. Aten Primaria 2002; 30: 112-118.

5. Plasencia A. Bases epidemiológicas para las políticas de prevención de los accidentes de tráfico relacionados con el alcohol en España. Adicciones 1999; 11: 229-236.

6. Fierro I, OchoA R, Yáñez JL, Valderrama JC, ÁlVAREZ FJ. Mortalidad y mortalidad prematura relacionadas con el consumo de alcohol en España entre 1999 y 2004. Med Clin 2008; 131: 10-13.

7. Calafat A, Adrover D, Juan M, Blay NT. Relación del consumo de alcohol y drogas de los jóvenes españoles con la siniestralidad vial durante la vida recreativa nocturna en tres comunidades autónomas en 2007. Rev Esp Salud Pública 2008; 82: 323-331.

8. Green J, Plant MA. Bad bars: A review of risk factors. J Subst Use 2007; 12: 157-189.

9. Rodríguez-Martos A. Prevención de lesiones atribuibles al alcohol en el marco de una política de reducción de daños. Trastornos Adictivos 2002; 4: 95-108.

10. Organización Mundial de la Salud. Estrategia mundial para reducir el consumo nocivo de alcohol. Laussane: Organización Mundial de la Salud 2010.

11. Goodsite B, Klear L, Rosenberg H. The impact of behavioral signs of intoxication on bartender service. Drugs 2008; 15: 545-551.
12. Shults RA, Elder RW, Sleet DA, Nichols JL, Alao MO, CARANDE-Kulis VG et al. Reviews of evidence regarding interventions to reduce alcohol-impaired driving. Am J Prev Med 2001; 21: 66-88.

13. Rodríguez-Martos A, García C, Durán A. Evaluación de un programa piloto de formación en dispensación responsable de bebidas alcohólicas. Trastornos Adictivos 2004; 6: 240 247.

14. Vidal A, Tomás S, Agullar R, Samper T, Zarza MJ, Aguilar J. Las cenas navideñas de empresa. ¿Un modelo de consumo recreativo de alcohol y otras drogas? Adicciones 2009; 21: 133142.

15. Mosher JF, Toomey TL, Good C, Harwood E, WAGENAAR AC. State laws mandating or promoting training programs for alcohol servers and establishment managers: an assessment of statutory and administrative procedures. J Public Health Policy 2002; 23: 90-113.

16. KeR K, Chinnock P. Interventions in the alcohol server setting for preventing injuries. Cochrane Database Syst Rev 2008; 16(3): CD005244.

17. Calafat A. Estrategias preventivas del abuso de alcohol. Adicciones 2002; 14: 317-335.

18. López-GoÑI JJ, Fernández-Montalvo J, Menéndez JC, Yudego F, García AR, Esarte S. Group and individual change in the treatment of drug addictions: A follow-up study in therapeutic communities. Span J Psychol 2010; 13: 906-913.

19. Terradillos J, Arteaga A. Cuestionario de Conocimientos sobre el Alcohol. Pamplona: Fundación Proyecto Hombre de Navarra 2006.

20. Rodríguez-Martos A, Gual A, Llopis JJ. La «unidad de bebida estándar" como registro simplificado del consumo de bebidas alcohólicas y su determinación en España. Med Clin 1999; 112: 446-450.

21. Martín A, Rojano P. Conceptos y anamnesis del consumo de alcohol en la consulta de Atención Primaria. Medicina General 2000; 29: 957-962.

22. Mora-Ríos J, Natera G, Juárez F. Expectativas relacionadas con el alcohol en la predicción del abuso en el consumo en jóvenes. Salud Mental 2005; 28: 82-90.

23. Pérez N, Milara J, Soler E, Ferrando R, Caja M, ROMERo R. Transcendencia del cocaetileno en el consumo combinado de etanol y cocaína. Rev Esp Drogodepend 2006; 31: 254-270.

24. Blanco P, Sirvent C. Psicopatología asociada al consumo de cocaína y alcohol. Rev Esp Drogodepend 2006; 31: 324-344. 